\title{
Subject Index to Volume 32
}

Absorptiometry, dual energy $\mathrm{x}$-ray, bone mineral content measurement, neonate, 77

Acetylcholine, coronary vasoconstriction induced by, neonatal pig, 236

Aciduria, 3-methylglutaconic, mitochondrial ATP-synthase deficiency in child with, 731

Actin, comparative polymerization, adult and neonatal bovine neutrophils, 509

Adenosine triphosphate, -sensitive potassium channels, neonatal and adult ventricular myocytes, rabbit, 230

Adipose tissue, development, thyroxine role, fetal pig, 204

$\beta$-Adrenergic receptor, characterization, human fetal lung type II cells, 350

Alae nasi, activation during oral feeding, preterm infants, 679

American Pediatric Society, 1992 presidential address, 373

Amino acid, comparison, protein turnover rates in neonates receiving parenteral nutrition, 447

Aminophylline, effect on diaphragmatic fatigue, piglet, 580

Antibody

human repertoire, development of, 257

maternal deficiency, antiidiotypic antibody induction in offspring, 1.50

non-maternal, offspring of mothers with antibody deficiencies, 150

Antigen, early dietary, development of gut mucosal barrier delayed by, preweaning rat, 301

Apolipoprotein, synthesis, intestinal explants, newborn piglet, 553

Arousal response, decrement after hypoxemia exposure, naloxone effect, lamb, 222

Arterial oxygen, tension threshold range arousal onset and breathing, fetal sheep, 342

ATP-synthase, mitochondrial deficiency, in child with 3-methylglutaconic aciduria, 731

Atrioventricular node, propafenone effects, rabbit, 658

Auditory brainstem response

amplitude changes, effect on neuron-specific enolase levels, piglet, 524

in transient neonatal hypothyroxinemia, preterm infants, 530

Betamethasone/thyrotropin releasing hormone, maternal administration, somatosensory evoked potentials after, preterm newborns, 212

Bilirubin, encephalopathy, neuron-specific enolase in, piglet, 524

Blood, viscosity, determination, preterm and full-term neonates, 97

Blood flow

cerebral

response to hypertension, phenobarbital-treated newborn pig, 103

response to hypocarbia in postasphyxia newborn lambs, 537

Blood pressure

measurement, random-zero sphygmoma- nometer, 243

oxygen supplementation effect, hypoxic newborn pig, 107

Bone mineral content, measurement, dual energy $\mathrm{x}$-ray absorptiometry, neonate, 77

Brain

capillary network, quantitative determination of morphometric indices, newborn pig, 542

cerebral oxidative metabolism, during hypothermia and circulatory arrest, newborn dog, 547

effect of postischemic hyperglycemia, neonatal rat, 489

heme oxygenase-2 protein activity, tin-protoporphyrin effects, rat, 324

phenobarbital effects, during hypertension and hypoxia, newborn pig, 103

quinolinate measurement, rat model of congenital hyperammonemia, 483

Brain damage, hypoxic, oxygen supplementation effect, newborn pig, 107

Breath test, noninvasive, starch digestion, cystic fibrosis patients, 45

Bronchopulmonary dysplasia, loaded breathing, respiratory drive and adaptation to, 356

Brush-border membrane vesicles, carrier-mediated $\beta$-D-hydroxybutyrate transport in, rat placenta, 317

Caffeine

during lactational period, cardiac effects, rat, 330

effect on diaphragmatic fatigue, piglet, 580

Capillary, total and perfused, quantitative determination of morphometric indices, newborn pig brain, 542

Carbohydrate, myocardial uptake, shunt lambs, 27

Cardiology, research, review of submitted abstracts, 1990, 10

Cardiovascular system, neonatal adaptation, in situ morphology, rat, 386

Carnitine, enterohepatic distribution, developing piglet, 312

$\mathrm{CD} 34^{+}$progenitor cells, isolation and separation, umbilical cord blood, 277

Cell injury, by reactive oxygen metabolites, differential sensitivity to, fibroblasts and endothelial cells, 654

Cerebrospinal fluid, quinolinate measurement, rat model of congenital hyperammonemia, 483

Cerebrovascular reactivity, alterations, after positive pressure ventilation, newborn pig, 114

Ceroid lipofuscinose, neuronal, molecular genetics, 645

Chemoreceptor, arterial peripheral, response time, preterm infants, 226

Chest wall, instability, inspiratory resistive loading effect, preterm infant, 589

Cholesteryl ester storage disease, lovastatin safety and efficacy, 559

Chondrocyte, epiphyseal, somatostatin effects, 571

Chondrodysplasia punctata, phytanic acid $\alpha$ oxidation in, 566
Circulatory arrest, hypothermic, cerebral oxidative metabolism during, newborn dog, 547

L-Citrulline, transport, across rat intestine, 472

Copper, intake and status, very low birth weight infants during first year, 183

Corticosterone, dietary, role, neonatal macromolecular absorption, rat, 50

Corticotropin-releasing hormone, stimulation test, nocturnal cortisol levels and 64

Cortisol, nocturnal levels, corticotropin-releasing hormone stimulation test, 64

Cyclosporin A, taurine transport impaired by, human placental cell line, 125

Cystic fibrosis

genotype/phenotype association, mutation analysis, 175

pancreatic-sufficient, exocrine pancreatic function decline in patients with, 179

starch digestion, noninvasive breath test for, 45

Cystine, intracellular loading, proximal tubule respiratory dysfunction caused by, rabbit, 710

Cytotoxicity, monosaturated and polyunsaturated fatty acids, cultured hamster cells, 366

Dehydration, hypernatremic, cerebral taurine transport and, rat, 118

Dexamethasone

combined with TRH, prenatal, hyperoxia survival shortened by, rat, 407

prenatal treatment, response to hyperoxic challenge after, newborn rat, 215

synergistic effects of thyroxine and, enzyme ontogeny, rat small intestine, 306

Diaphragm

fatigue, methylxanthine effect, piglet, 580

nitrofen-induced hernia, oxygen defense mechanisms, rat, 394

Diarrhea, circulating antibody secreting cell response, enhancement, Lactobacillus strain, 141

Dichloroethylene, cardiac teratogenicity, chick model, 23

Dihydropteridine reductase, atypical deficiency, neurochemical evaluation and mutation detection, 726

Docosahexaenoic acid, status, breast-fed vs. oil-containing formula-fed term infants, 683

Dorsal root ganglia, latency in vitro of varicella-zoster virus in cells derived from, 699

Down syndrome, thymocyte proliferation, regulation by tumor necrosis factor $-\alpha$ and interferon- $\gamma, 269$

Duchenne muscular dystrophy, revertant frequency, mouse model, 128

Ductus venosus, ultrasonic assessment, human fetus, 338

Embryo, early cardiac development, dynamic atrial dimension during, chick, 333 
Encephalopathy

bilirubin, neuron-specific enolase in, piglet, 524

neuronal ceroid lipofuscinosis, molecular genetics of, 645

Endothelial cells, antioxidant defense mechanisms, role of glutathione redox cycle and catalase, 360

Energy expenditure

sleep organization and, breast-fed and formula-fed infants, 514

theophylline-treated preterm infants, 693

Enolase, neuron-specific, in bilirubin encephalopathy, piglet, 524

Enzyme

branching, inherited deficiency, feline glycogen storage disease type IV, 719

antioxidant, role, diaphragmatic hernia, rat, 394

ontogeny, synergistic effects of thyroxine and dexamethasone on, rat small intestine, 306

Eosinophil

activation, respiratory syncytial virus, 160

L-selectin expression and regulation, human adults and neonates, 465

Epithelium, hyperoxia-induced injury, prostaglandin $E_{2}$ effect, rabbit trachea, 87

Erythrocyte

aging process, hemoglobin $\mathrm{F}$ and, neonate, 165

membrane, mechanical fragility, neonates and adults, 92

oxidative stress, glutathione recycling during, neonate, 399

Erythroid progenitor cells, enhanced response to hGH and IGF-1, children with insufficient $\mathrm{GH}$ secretion, 282

Ethanol, prenatal exposure, effect on postnatal maturation of small intestine and liver, rat, 574

European Society for Pediatric Research, abstracts of 1992 annual meeting, 609

Fatty acids: see also Free fatty acids monosaturated and polyunsaturated, effect on oxygen toxicity, cultured hamster cells, 366

myocardial uptake, shunt lambs, 27

FCRIII, increasing receptor production, during late pregnancy and after preterm birth, 505

Feeding, cardiovascular responses to, newborn piglet, 33

Fetus

adipose tissue and skin differentiation, thyroxine role, pig, 204

cultured epiphyseal chondrocytes, somatostatin effects, 571

dorsal root ganglia, in vitro latency of varicella-zoster virus in cells from, 699

fibroblast, binding of placental lactogen and $\mathrm{GH}$ to, sheep, 200

GH-binding proteins, serum levels, 69

hematopoiesis, alterations, maternal HIV infection, 155

hepatic lipid metabolism, guinea pig model of intrauterine growth retardation, 441

hepatocyte, glycogenic response to glucose, rat, 714

in situ morphology of foramen ovale, rat, 669

intestinal mucosal immunity, comparison, newborn, 145

lung type II cells, $\beta$-adrenergic receptor characterization in, 350

morphology of ductus venosus, rat, 386 onset of arousal and breathing, arterial oxygen tension threshold range for, sheep, 342

subdiaphragmatic area, venous distribution, ultrasonic visualization, 338

thoracic duct lymph flow, outflow pressure effect, sheep, 585

tissue growth and glucose utilization, effect of maternal hypoglycemia, rat, 436

Fibroblasts

binding of placental lactogen and GH to, fetal sheep, 200

sensitivity to reactive oxygen metabolites, comparison, endothelial cells, 654

Foramen ovale, in situ morphology, fetal and neonatal rat, 669

Free fatty acids, hepatic metabolism, guinea pig model of intrauterine growth retardation, 441

Galactose, intolerance, magnetic resonance spectroscopy study, human and rat liver, 39

Gaucher disease, neonatal, distinct phenotype of, 494

Genetics

molecular, neuronal ceroid lipofuscinosis, 645

mutation detection, atypical forms of dihydropteridine reductase deficiency, 726

new OTC gene mutations, 600

ontogeny of kininogen gene expression, rat, 649

pulmonary metallothionein gene expression, late gestational lamb, 424

targeted disruption of glucocerebrosidase gene, mouse model of Gaucher disease, 494

Glucagon, role, regulation of enterohepatic carnitine distribution, piglet, 312

Glucocerebrosidase gene, targeted disruption, mouse model of Gaucher disease, 494

Glucose

glycogenic response, fetal and adult rat hepatocyte, 714

production, theophylline-treated preterm infants, 693

utilization, fetal, effect of maternal hypoglycemia, rat, 436

Glutathione, recycling, during oxidative stress, neonatal erythrocytes, 399

Glutathione peroxidase, levels, premature infants, low selenium community, 189

Glycogenesis, regulation by medium glucose, fetal and adult rat hepatocyte, 714

Glycogen storage disease, type IV, clinical, pathologic, biochemical and genetic characteristics, cat, 719

Growth and development

adipose tissue and skin differentiation, thyroxine influence, fetal pig, 204

anion transport in cardiac sarcoplasmic reticulum, fetal and adult rabbit, 664

ATP-sensitive potassium channels, ventricular myocytes, neonatal and adult rabbit, 230

comparative actin polymerization, neonatal and adult bovine neutrophils, 509

dietary protein effect, recovering shigellosis patients, 689

differential developmental expression of kininogen genes, rat, 649

erythrocyte membrane fragility, neonates and adults, 92

fate of the endocardial cushions, developing chick heart, 390 glycogenesis, regulation, fetal and adult rat hepatocyte, 714

gut mucosal barrier, effect of early dietary antigens, preweaning rat, 301

inspiratory resistive loading effects, preterm and full-term infants, 589

intestinal mucosal immunity, fetus and newborn, 145

jejunal closure, dietary corticosterone role, 50

pulmonary metallothionein gene expression, late gestational lamb, 424

regulation of L-selectin on eosinophils, human adults and neonates, 465

renal phosphate transport maturation, early weaning and, rat, 704

serum GH-binding proteins, human fetus and infant, 69

$T$ cell functional changes, ontogeny of cellular immunity and, 81

Growth factors, hematopoietic, response of $\mathrm{CD} 34^{+}$progenitor cells to, umbilical cord blood, 277

Growth hormone

binding, fetal sheep fibroblasts, 200

insufficient secretion, erythropoietic effects of hGH and IGF-I in children with, 282

pulsatile, deconvolution analysis, neonate, 286

urinary measurements, normal and short children, 73

Growth retardation

intrauterine

after uterine artery ligation, rat, 291

hepatic lipid metabolism, guinea pig, 441

Gut, mucosal immunity, development, fetus and neonate, 145

Haemophilus influenzae $\mathrm{b}$ polysaccharide, vaccine-induced human antibody responses, immunodeficient mice, 132

Haemophilus influenzae type $\mathrm{b}$, human im-

Heart mune response to, 257

anion transport in sarcoplasmic reticulum, fetal and adult rabbit, 664

dichloroethylene teratogenicity, chick model, 23

early development, dynamic atrial dimension and function during, chick embryo, 333

fate of the atrioventricular endocardial cushions, developing chick, 390

propafenone effects, rabbit atrioventricular node, 658

vasoconstriction, acetylcholine-induced, neonatal pig, 236

ventricular myocytes, ATP-sensitive potassium channels, neonatal and adult rabbit, 230

zinc and metallothionein levels, effect of dietary caffeine, neonatal rat, 330

Hematocrit, optimal, determination, preterm and full-term neonates, 97

Hematopoiesis, alterations, fetus, HIV infected mothers, 155

Heme oxygenase isoforms, tin-protoporphyrin effect, rat brain, 324

Hemoglobin F, oxidation, aging of neonatal red blood cells and, 165

Hepatitis $B$ virus, $S$ gene mutations, mother to child transmission of hepatitis B related to, 264

Hepatocyte, glycogenic response to glucose, fetal and adult rat, 714

HIV: see Human immunodeficiency virus 
Howland Award

acceptance by Gilbert B. Forbes, 502

presentation, 499

Human immunodeficiency virus, maternal infections, fetal hematopoiesis and, 155

Hydrocarbon, halogenated, dichloroethylene-related cardiac teratogenicity, chick, 23

$\beta$-D-Hydroxybutyrate, $\quad$ carrier-mediated transport, brush-border membrane vesicles, rat placenta, 317

Hyperammonemia, congenital, quinolinate measurement, rat brain and CSF, 483

Hyperglycemia

maternal, fetal rat tissue growth and glucose utilization, 436

postischemic, effect on neonatal rat brain, 489

Hypernatremia, cerebral taurine transport, rat, 118

Hyperoxia

antioxidant enzyme response, after prenatal TRH or TRH plus dexamethasone, rat, 407

-induced injury, prostaglandin $\mathrm{E}_{2}$ effect, rabbit tracheal epithelial cells, 87

Hypertension, with and without hypoxia, cerebral blood flow response, phenobarbital-treated newborn pig, 103

Hypocarbia, response of cerebral circulation to, postasphyxia newborn lambs, 537

Hypothermia, cerebral oxidative metabolism during, newborn dog, 547

Hypothyroxinemia, transient neonatal, auditory brainstem evoked response in, 530

Hypoxanthine, plasma levels, after oxygen supplementation, hypoxic newborn pig, 107

Hypoxemia, arousal response decrement after, naloxone effect, lamb, 222

Hypoxia

phenobarbital effect, hypertensive newborn pig, 103

resuscitation, room air versus $100 \%$ oxygen, newborn pig, 107

ventilatory response, small preterm infants, 403

IFN: see Interferon

Immune system, development of human antibody repertoire, 257

Immunization, hepatitis $B$ virus, mother to child transmission of $\mathrm{HBV}$ despite, 264

Immunoglobulin, development of human antibody repertoire, 257

Infant

GH-binding proteins, serum levels, 69

metabolism and water loss, effect of mild cold stress on, 417

preterm

activation of plasma kallikrein-kinin system in respiratory distress syndrome, 431

alae nasi activation during oral feeding, 679

auditory brainstem evoked response in neonatal hypothyroxinemia, 530

effect of prenatal thyrotropin-releasing hormone treatment, 673

effects of inspiratory resistive loading, 589

elevated GH secretory rate, 286

parenteral nutrition, protein turnover changes after, 447 reduced membrane-associated FcRIII expression, 505

response time, peripheral chemoreceptors, 226

selenium and glutathione peroxidase levels, low selenium community, 189

somatosensory evoked potentials after fetal betamethasone/thyrotropin releasing hormone therapy, 212

theophylline effect on energy expenditure, lipolysis, and glucose production, 693

ventilatory response to hypoxia, 403

very low birth weight, copper status during first year, 183

Infant feeding/nutrition

docosahexaenoic acid status, breast-fed versus oil-containing formula-fed term infants, 683

oral, alae nasi activation during, preterm infants, 679

sleep organization influenced by, breastfed and formula-fed infants, 514

Infant formula

oil-containing, docosahexaenoic acid status in term infants receiving, 683

polyamine concentration, 58

Inferior vena cava, ultrasonic assessment, human fetus, 338

Insulin, role, regulation of enterohepatic carnitine distribution, piglet, 312

Insulin-like growth factor, decreased activity, intrauterine growth retardation related to, rat, 291

Insulin-like growth factor-I

destripeptide, isolation and characterization, bovine somatotropin- treated cows, 296

erythroid progenitor cell response to, children with insufficient GH secretion, 282

receptor, autoantibodies to, low prevalence, short stature children, 455

Interferon $\gamma$

regulation of thymocyte proliferation by Down syndrome, 269

role, newborn host defense against Listeria monocytogenes, 460

Interleukin-4, human thymocyte proliferation induced by, Down syndrome, 269

Intestinal mucosa, immunity development, fetus and newborn, 145

Jejunum, closure, role of dietary corticosterone, neonatal rat, 50

Kallikrein-kinin, plasma system, activation, respiratory distress syndrome, 431

Ketone, myocardial uptake, shunt lambs, 27

Kidney

phosphate transport maturation, early weaning effects, rat, 704

renal tubular epithelial cells, antioxidant defense mechanisms, 360

Kininogen gene, differential developmental expression, rat liver, 649

Lactobacillus, effect on intestinal immune response, human diarrhea, 141

Lipolysis, theophylline-treated preterm infants, 693

Listerial monocytogenes, newborn host defense against, role of TNF- $\alpha$ and IFN$\gamma, 460$

Liver

differential developmental expression of kininogen genes, rat, 649

galactose intolerance, magnetic resonance spectroscopic study, human and rat
39

lipid metabolism by, guinea pig model of intrauterine growth retardation, 441

postnatal maturation, prenatal ethanol exposure effect, rat, 574

Local anesthesia, eutectic mixture, effectiveness, venipuncture patients, 520

Lovastatin, safety and efficacy, pediatric cholesteryl ester storage disease, 559

Lung

adaptation, loaded breathing in bronchopulmonary dysplasia, 356

antioxidant enzyme, role, diaphragmatic hernia, rat, 394

immediate effects of surfactant, preterm lamb, 595

pulmonary metallothionein genes, expression, late gestational lamb, 424

type II cells, $\beta$-adrenergic receptor characterization, human fetus, 350

Luteinizing hormone, pulsatile and sexually dimorphic secretion of, postnatal day 1, 605

Lymph, flow rate, outflow pressure effect, fetal sheep, 585

Malaria, design of conjugate vaccines for, 376

Meningitis, bacterial, design of conjugate vaccines for, 376

Meperidine, maternal administration, neonatal pattern of breathing after, 412

Metabolic rate, environmental temperature and, 417

Metallothionein

effect of dietary caffeine, neonatal rat heart, 330

pulmonary genes, expression of, late gestational lamb, 424

Methylxanthines, effect on diaphragmatic fatigue, piglet, 580

bovine somatotropin-treated cows, isolation of destripeptide IGF-I from, 296

human, polyamine concentration, 58

rat, polyamine concentration, 58

Mitochondria

ATP-synthase deficiency, child with 3methylglutaconic aciduria, 731

myopathy, permeabilized muscle fibers, 17

Muscle fiber, permeabilized, mitochondria myopathy studies, 17

Myocardium, substrate uptake, shunt lambs, 27

Naloxone, effect on arousal response decrement, after hypoxemia exposure during sleep, lamb, 222

Neisseria gonorrhoeae, design of conjugate vaccine for, 376

Neonate

antiidiotypic antibody induction, 150

apolipoprotein synthesis, piglet intestinal explants, 553

autonomic reactivity, cardiovascular responses to feeding, newborn pig, 33

blood viscosity and optimal hematocrit, preterm and full-term subjects, 97

bone mineral content, measurement, dual energy $\mathrm{x}$-ray absorptiometry, 77

brain capillary network, quantitative determination of morphometric indices, pig, 542

cardiac effects of dietary caffeine during lactation, rat, 330

CD $34^{+}$cord blood cells, stem cell factor and PIXY321 effects, 277

cerebral blood flow, response to hypocarbia after asphyxia, lamb, 537

erythrocytes 
glutathione recycling during oxidative stress in, 399

membrane fragility, comparison, adults, 92

feeding, cardiovascular responses, piglet,

Gaucher disease, distinct phenotype analogous to a mouse model, 494

host defense against Listeria monocytogenes, role of TNF- $\alpha$ and IFN- $\gamma, 460$

hyperoxic challenge

prenatal dexamethasone effect, rat, 215

prenatal TRH or TRH plus dexamethasone effect, rat, 407

hypothermia and circulatory arrest, cerebral oxidative metabolism during, dog, 547

hypoxic, oxygen supplementation, pig, 107

in situ morphology of foramen ovale, rat, 669

intestinal mucosal immunity, comparison, fetus, 145

ischemia, effect of hyperglycemia after, rat brain, 489

luteinizing hormone secretion, dynamics of, postnatal day 1, 605

macromolecular absorption, dietary corticosterone role, rat, 50

mitochondrial myopathy studies, permeabilized muscle fibers, 17

modified natural surfactant treatment, cerebral hemodynamic effects, 532

morphology of ductus venosus, rat, 386

pattern of breathing, after maternal meperidine, 412

positive pressure ventilation, cerebrovascular reactivity alterations after, pig, 114

pulsatile GH secretion, deconvolution analysis of, 286

red blood cell aging process, hemoglobin $\mathrm{F}$ oxidation and, 165

regulation of enterohepatic carnitine distribution, glucagon and insulin role, piglet, 312

Neutrophil

comparative actin polymerization, neonatal and adult, 509

reduced FcRIII expression by, preterm neonate, 505

Nutrition

effect of early dietary antigens, gut mucosal barrier, preweaning rat, 301

high-protein diet effect, effect on catch-up growth, shigellosis patients, 689

research and training, NICHD plan for, 1

selenium supplementation, premature infants, low selenium community, 189

Ornithine transcarbamylase, new gene mutations, OTC-deficient patients, 600

OTC: see Ornithine transcarbamylase

Oxidant stress, role of glutathione redox cycle and catalase, endothelial and renal tu-

Oxygen bular epithelial cells, 360

consumption, cystine-loaded proximal tubules, rabbit, 710

reactive metabolites, differential sensitivity, human fibroblasts and endothelial cells, 654

response time, peripheral chemoreceptors, preterm infants, 226

supplementation, reoxygenation of hypoxic newborn, newborn pig, 107

toxicity

prenatal dexamethasone treatment effect, rat, 215 fatty acids effects, cultured hamster cells, 366

Pain, perception, effectiveness of eutectic mixture of local anesthetics and, venipuncture patients, 520

Pancreas, exocrine function, decline, cystic fibrosis patients with pancreatic sufficiency, 179

Parenteral nutrition, neonatal, protein turnover rates with two amino acid solutions, 447

PDC: see Pyruvate dehydrogenase

Phenobarbital, cerebral blood flow and, during hypertension and hypoxia, newborn pig, 103

Phosphate, renal transport maturation, effects of early weaning, rat, 704

Phytanic acid, $\alpha$-hydroxylation of, Refsum's disease and chondrodysplasia punctata, 566

Placenta

carrier-mediated $\quad \beta$-D-hydroxybutyrate transport, rat, 317

perfused, retinol transfer and metabolism by, 195

taurine transport, impairment by cyclosporin A, 125

Placental lactogen, binding, fetal sheep fibroblasts, 200

Polyamine, concentration, rat milk, human milk, infant formulas, 58

Polysaccharide, vaccine-induced human antibody responses, immunodeficient mice, 132

Potassium, ATP-sensitive channels, neonatal and adult ventricular myocytes, rabbit, 230

Prematurity, immediate pulmonary effects of surfactant, preterm lamb, 595

Prolidase, deficiency, cultured human fibroblasts, 479

Propafenone, developmental cellular electrophysiologic effects, rabbit atrioventricular node, 658

Prostaglandin $E_{2}$, hyperoxia-induced injury and, rabbit tracheal epithelial cells, 87

Prostanoids, vasoactive, increased biosynthesis, Schönlein-Henoch purpura, 137

Protein

GH-binding, serum levels, human fetus and infant, 69

-rich diet, effect on catch-up growth after shigellosis, 689

turnover rates, after parenteral nutrition introduction, preterm infants, 447

Proximal tubule, cystine-loaded, respiratory dysfunction caused by, rabbit, 710

Pseudomonas aeruginosa, design of conjugate vaccine for, 376

Pyruvate dehydrogenase, mutation, variable expression of PDC deficiency caused by, 169

Quinolinate, brain and CSF levels, rat model of congenital hyperammonemia, 483

Refsum's disease, phytanic acid $\alpha$-oxidation in, 566

Respiration

during active and quiet sleep, neonate, after maternal meperidine, 412

onset, arterial oxygen tension threshold range for, fetal sheep, 342

Respiratory distress syndrome, activation of plasma kallikrein-kinin system in, 431

Respiratory syncytial virus, eosinophil activation by, 160
Retinol, transfer and metabolism, perfused human placenta, 195

Revertant, frequency, Duchenne muscular dystrophy, mouse model, 128

Rose's tape, evaluation, blood pressure measurement accuracy, 243

Sarcoplasmic reticulum, anion transport, fetal and adult rabbit heart, 664

Schönlein-Henoch purpura, biosynthesis of vasoactive prostanoids in, 137

L-Selectin, expression and regulation, eosinophils from human adults and neonates, 465

Selenium, levels, premature infants, low selenium community, 189

Shigellosis, catch-up growth after, proteinrich diet effect, 689

Short stature

IGF-I receptor autoantibodies in children with, 455

urinary GH measurements, clinical usefulness, 73

Shunt, aortopulmonary, myocardial substrate uptake in lambs with, 27

Sleep organization, energy expenditure and, breast-fed and formula-fed infants, 514

Sleep state, environmental temperature and, 417

Small intestine

apolipoprotein synthesis, newborn piglet explants, 553

L-citrulline transport across, rat, 472

enzyme ontogeny, synergistic effects of thyroxine and dexamethasone on, rat, 306

postnatal maturation, prenatal ethanol exposure effect, rat, 574

Sociedad Latinoamericana de Endocrinologia Pediatrica, abstracts from 5th annual meeting, 249

Society for Pediatric Research, 1992 presidential address, 641

Somatomedin, isolation, bovine somatotropin-treated cows milk, 296

Somatosensory evoked potentials, effect of prenatal betamethasone/thyrotropin, preterm newborns, 212

Somatostatin, effects on cultured human fetal epiphyseal chondrocytes, 57.

Spectroscopy

magnetic resonance, galactose intolerance studies, human and rat liver, 39

near infrared, effects of surfactant treatment studied by, 532

Sphygmomanometer, random-zero, blood pressure measurement, 243

Starch, digestion, ${ }^{13} \mathrm{C}$ breath test for, cystic fibrosis patients, 45

Stem cell factor, effect on myeloid progenitor formation, human umbilical cord blood, 277

Stress, response patterns, measurement, normal children, 64

Surfactant

modified natural, cerebral hemodynamic effects, neonates, 532

natural, lung volume and ventilation effects, preterm lamb, 595

Taurine

cerebral transport, in hypernatremic dehydration, rat, 118

transport, cyclosporin A effect, human placental cell line, 125

$T$ cell, functional changes, ontogeny of cellular immunity and, 81

Temperature, environmental, metabolic rate, 


\section{SUBJECT INDEX TO VOLUME 32}

sleep state, and water loss related to, 417

Theophylline, effect on energy expenditure, lipolysis, and glucose production, preterm infants, 693

Thoracic duct, lymph flow rate, outflow pressure effect, fetal sheep, 585

Thyroid function, after prenatal thyrotropinreleasing hormone, preterm infants and their mothers, 673

Thyroid releasing hormone, prenatal, effect on hyperoxia survival, neonatal rat, 407

Thyrotropin-releasing hormone, prenatal treatment, effects on premature infants and their mothers, 673

Thyroxine

role, differentiation of adipose tissue and skin, fetal pig, 204

synergistic effects of dexamethasone and, enzyme ontogeny, rat small intestine, 306

Tin-protoporphyrin, effects on heme oxygenase- 2 protein activity, rat brain, 324
Tissue growth, effect of maternal hypoglycemia, fetal rat, 436

TNF: see Tumor necrosis factor

Trachea, epithelial cells, hyperoxia-induced injury, prostaglandin $\mathrm{E}_{2}$ effect, rabbit, 87

TRH: see Thyroid releasing hormone

Triacylglycerols, hepatic metabolism, guinea pig model of intrauterine growth retardation, 441

Tumor necrosis factor- $\alpha$ regulation of thymocyte proliferation by, Down syndrome, 269

role, newborn host defense against Listeria monocytogenes, 460

Ultrasound, assessment, fetal subdiaphrag-

Uterine artery, ligation, IGF regulation and intrauterine growth retardation after, rat, 291

Vaccine

conjugate, rational design of, 376

hepatitis B virus, escaped mutants, 264 matic vestibulum, 338
Varicella-Zoster virus, latency in vitro, fetal dorsal root ganglia cells, 699

Venipuncture, determinants of pain perception in children undergoing, 520

Ventilation

artificial, effect on antioxidant defense mechanisms, congenital diaphragmatic hernia, rat, 394

effect of inspiratory resistive loading, preterm and full-term infants, 589

homogeneity, effect of natural surfactant, preterm lamb, 595

positive pressure, cerebrovascular reactivity alterations after, 114

response to hypoxia, small preterm infants, 403

Water loss, evaporative, environmental temperature and, 417

Weaning, early, effect on phosphate transport maturation, rat kidney, 704

Zinc, effect of dietary caffeine, neonatal rat heart, 330 